\title{
Characterization of Cardiac Dynamics from Locally Topological Considerations
}

\author{
Victor F. Dailyudenko \\ Institute of Informatics Problems NAS of Belarus, \\ Surganov St. 6, 220012, Minsk, Belarus \\ selforg@newman. bas-net.by
}

\begin{abstract}
Evolution of cardiac activity is investigated by means of methods of nonlinear dynamics, namely the method of temporal localization on the attractor reconstructed from electrocardiogram (ECG) signal is proposed for this purpose. Convergence for the function of topological instability at changing dimensionality is proved both theoretically and numerically, independently on personal features of subjects in the latter case, that provides the opportunity to estimate the complexity of cardiac dynamics. In contrast, that instability function normalized by its average displays different kind of behaviour that somewhat differs for various persons and reflects their individual features.
\end{abstract}

\section{Introduction}

The computational complexity of topological algorithms being applied for cardiovascular dynamics investigation makes these algorithms rather cumbersome from standpoint of their computer time and the quantity of required experimental data $N$ [1]. Therefore, the total time of observation and diagnosis process becomes rather long that may result in difficulties in clinical practice. So, in this paper we develop the topological method based on temporal locality approach. In comparison with the most conventional methods of nonlinear analysis based on spatial localization [1-4], the developed method allows reduction of $N$ and computation time as well as is insensible to growing $m$ on these characteristics.

\section{The Algorithm for Exploration of Topological Instability}

The method of delayed coordinates (affirmed mathematically by Takens [5]) for reconstruction of phase trajectories forming an attractor $R_{T}^{m}$ is given by $[1,2,4-5]$

$$
\vec{x}_{i}^{(m)}=\left(\eta_{i}, \eta_{i+p}, \ldots, \eta_{i+(m-1) p}\right),
$$

where $\eta(i \Delta t)=\eta_{i}, i=1,2, \ldots, N$ is a time series (TS) of a kinetic variable measured with a fixed time interval $\Delta t, \tau=p \Delta t$ is the delay time, $\mathrm{p}$ is an integer. The points $\vec{x}_{i}^{(m)} \subset R^{m}, R^{m}$ is an Euclidean phase space with a dimension $m, i=1,2, \ldots, L^{(p, m)}$, 
the common quantity of the attractor points is given by $L^{(p, m)}=N-p(m-1)$. In accordance with (1), phase trajectories forming the attractor $R_{T}^{m}$ can be represented as a superposition of $p$ rarefied sequences $\mathrm{X}_{1}, \mathrm{X}_{2}, \ldots, \mathrm{X}_{p}$ shifted by one sample with respect to each other, those are defined as $\mathrm{X}_{s}=\left\{\vec{x}_{s+p(k-1)}^{(m)}\right\}_{k=1}^{L_{s}^{(p, m)}}$.

As it was recently shown, rarefying on attractor points is reasonable for numerical simulation of fractal-topological analysis [4]. Otherwise, using points that are too close together in time leads to essential underestimates of the dimension, i.e. to aggravating accuracy of the topological analysis. So, we also implement temporal rarefying of phase trajectories for creating a subset of points with decorrelated components resulting in essentially random distribution in the embedding space. It is attained by the approach that is realized in the most convenient way, namely we use only one $X_{s}$ for numerical experiments. That is constructed using the sequence $\Psi_{p}=\left\{\eta_{p}, \eta_{2 \cdot p}, \ldots, \eta_{N_{p}^{(p)} \cdot p}\right\}$, and rarefying is determined with $p=2$. Denoting components of $\Psi_{p}$ for brevity as $\Psi_{p}=\left\{\xi_{1}, \xi_{2}, \ldots, \xi_{N_{p}^{(p)}}\right\}$, we obtain that the terms of relative partition sequence $\left\{\mu_{j}^{(m)}\right\}$ constructed by means of segmentation of difference-quadratic TS are defined analogously [6] as follows

$$
\mu_{j}^{(m)}=\frac{\Delta \xi_{j+m}}{\hat{\sigma}_{j}^{(m)}}
$$

where $\hat{\sigma}_{j}^{(m)}=\sum_{i=0}^{m-1} \Delta \xi_{j+i}^{2}, \Delta \xi_{j}=\xi_{j+1}-\xi_{j}$. Similarly [6], introduce the following measure of topological instability:

$$
Z_{\mu}(m)=\sigma\left(\mu_{j}^{(m)}\right),
$$

where $\sigma\left(\mu_{j}^{(m)}\right)$ is the mean square variance, the averaging is made over $R_{T}^{m}$ points. For estimating the relative variance on $\left\{\mu_{j}^{(m)}\right\}$, introduce the following normalized instability function:

$$
\breve{Z}_{\mu}(m)=\frac{Z_{\mu}(m)}{\left\langle\mu_{j}^{(m)}\right\rangle}=\left(\frac{\left.\left\langle\mu_{j}^{(m)}\right)^{2}\right\rangle}{\left\langle\mu_{j}^{(m)}\right\rangle^{2}}-1\right)^{\frac{1}{2}} .
$$

\section{Numerical Simulations with ECG Signal}

In this work the digitized ECG TS $\zeta_{i}$ containing $\mathrm{N}=2500$ points is used, $\Delta t=2 m c$. The initial part of measured TS is shown in Fig. 1. The obtained TS is that of an adult healthy subject being under physical exercises. For decreasing linear autocorrelation 
effect and reduction of influence of low-frequency periodical component, we use difference TS $\eta_{i}=\varsigma_{i}-\varsigma_{i+1}$ instead of "raw" digitized ECG signal $\varsigma_{i}$ (as well as in [1]). The phase trajectories for three-dimensional attractors reconstructed from $\eta_{i}$ by (1) at $p=1$ and $p=20$ respectively are shown in Fig. 2, a) and b), the latter case reveals the effect of partial decorrelation (similarly [1]).

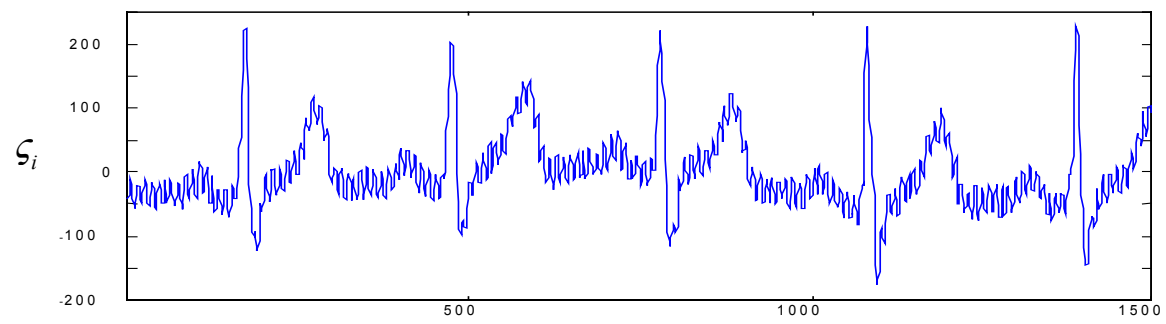

Fig. 1. The initial ECG signal taken out of the first database

For investigation of topological dependencies, we use three databases of ECG signals obtained from different groups of healthy adult subjects at the same conditions as for the ECG signal displayed in Fig. 1, the following additional normalization being used for scale unification:

$$
Y(m)=\frac{Z_{\mu}(m)}{Z_{\mu}(1)}, \quad \breve{Y}(m)=\frac{\check{Z}_{\mu}(m)}{\check{Z}_{\mu}(1)} .
$$
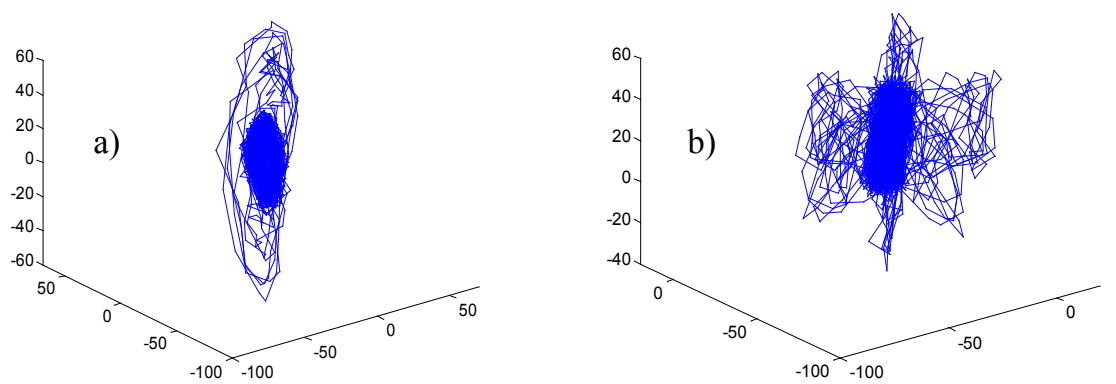

Fig. 2. Temporal evolution of cardiac activity represented through the phase trajectories reconstructed from the difference ECG TS: a) $p=1$; b) $p=20$

Calculated dependencies (5) are shown in Fig. 3, a) and b). These dependencies display sufficient convergence for $m \geq m_{0}$, i.e. $m_{0}$ is just the value of a dimension that provides preservation of topological structure of phase trajectories at enlarging dimensionality beyond $m_{0}$ and thus can be really considered as the minimal 
embedding dimension of the attractor. The convergence of $Y(m)$ is shown to be of the same character, independently on individual features of subjects, and one can conclude that $m_{0}=6$ is sufficient for optimal embedding of the attractor into Takens' phase space. On the other hand, the dependence $\breve{Y}(m)$ is approximately linear at $m \geq m_{0}$, but it differs for various persons with respect to average level of convergence and a slant angle. Evidently, it can provide some additional information concerning individual features of subjects that seems to be useful for the sake of early diagnosis.
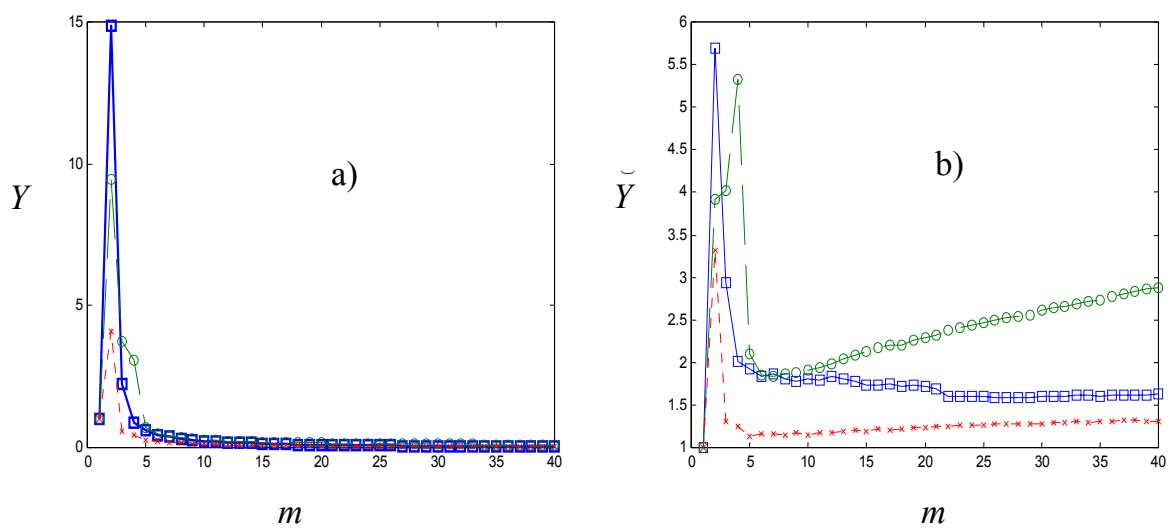

Fig. 3. The topological dependencies calculated with ECG TS from samples randomly chosen out of three different databases

\section{Conclusions}

The obtained results of $m_{0}$ determined on $Y(m)$ are in good coincidence with those obtained in [1] by the Grassberger - Procaccia algorithm (GPA), where slopes of the plots $\ln C(l)$ versus $\ln l$ appear to be same for $\mathrm{m} \geq 6$. It is worth to note that in GPA $\mathrm{N}=16000$ [1]; while in our experiments $\mathrm{N}=2500$ (moreover, the length of really used rarefied TS $N_{p}^{(p)}=1250$ ) that means the significant reduction of data.

\section{References}

1. Bezerianos, A., Bountis, T., Papaioannou, G., Polydoropoulos, P.: Nonlinear time series analysis of electrocardiograms. Chaos. 5 (1995) 95 - 101

2. Nonnenmacher, T.F., Losa, G.A., Wribel E.R. (editors): Fractals in Biology and Medicine, Birkhauser - Verlag, (1994)

3. Wang, J., Ning, X., Chen, Y.: Multifractal analysis of electronic cardiogram taken from healthy and unhealthy adult subjects. Physica A. 323 (2003) 561 - 568 
4. Albano, A.M., Passamante, A., Farrell, M.E.: Using higher-order correlations to define an embedding window. Physica D. 54 (1991) 85 - 97

5. Takens, F.: Detecting strange attractors in turbulence In: Dynamical Systems and Turbulence. Lecture Notes in Math., Springer, Berlin, 898 (1981) 366 - 381

6. Dailyudenko, V.F.: Nonlinear time series processing by means of ideal topological stabilization analysis and scaling properties investigation. In: Proc. of the SPIE's Conf. on Applications and Science of Computational Intelligence II (Apr. 1999, Orlando, Florida, USA) 3722 (1999) 108 - 119 